\title{
Quartiers, rues et espaces publics : éléments pour une histoire des Etudes Urbaines francophones
}

Yankel FIJALKOW avec la collaboration scientifique d'Amélie NICOLAS

\author{
(2) OpenEdition \\ 12 Journals \\ Édition électronique \\ URL : http://journals.openedition.org/eue/2144 \\ ISSN : 1916-4645

\section{Éditeur} \\ Institut national de Recherche scientifique Urbanisation Culture et Société
}

\section{Référence électronique}

Yankel FIJALKOW avec la collaboration scientifique d'Amélie NICOLAS, «Quartiers, rues et espaces publics : éléments pour une histoire des Etudes Urbaines francophones », Environnement Urbain / Urban Environment [En ligne], Volume 13 | 2018, mis en ligne le 03 juillet 2018, consulté le 13 septembre 2018. URL : http://journals.openedition.org/eue/2144

Ce document a été généré automatiquement le 13 septembre 2018.

(C) Institut National de Recherche Scientifique Centre Urbanisation Culture et Société 


\section{Quartiers, rues et espaces publics : éléments pour une histoire des Etudes Urbaines francophones}

Yankel FIJALKOW avec la collaboration scientifique d'Amélie NICOLAS

\section{Introduction}

1 À la confluence des sciences sociales de la ville et de l'urbanisme, les études urbaines constituent un champ de recherche composite issu, à la fois de la contribution des études sociales, historiques et géographiques à l'émergence du champ professionnel de l'urbanisme, et des enquêtes sociologiques et anthropologiques du début du XXème siècle sur le milieu urbain et ses modes de vie. En France, ce courant s'est d'abord constitué autour de la publication du premier numéro de la revue de l'Institut d'Urbanisme de Paris, La Vie Urbaine (1919), qui accueillit des auteurs comme Maurice Halbwachs et Raoul Blanchard, puis de la création par Chombart de Lauwe vers 1950 du Groupe d'ethnologie sociale inspiré par les recherches de l'Ecole de Chicago. Depuis le développement de la recherche urbaine au cours du XXème siècle, avec ses instituts, ses enseignements, ses centres de recherches, et ses revues, ce milieu intellectuel s'est développé. Il oppose moins des chercheurs et des praticiens, qu'une manière de qualifier et d'investiguer les rapports entre formes sociales et spatiales. Il associe de manière originale savoirs académiques et savoirs d'action. À ce titre, les monographies de villes et de quartiers, mobilisées dès l'origine des études urbaines, sont toujours d'actualité tant pour fonder une théorisation générale de la ville que pour répondre aux questions des professionnels de l'urbain. Elles convoquent des micros échelles comme le quartier et la rue; des notions comme celle d'espace public; des processus socio-économiques de production des espaces. Mais quels sens ces notions ont-elles aujourd'hui au sein de la communauté scientifique et pour les populations concernées? Comment ont-elles évolué selon les orientations des recherches et des politiques publiques? Comment se traduisent-elles dans les projets urbains? Dans quelle mesure l'usage de la langue française dans les 
études urbaines leur apporte une spécificité ? Sont-elles aujourd'hui en mesure de développer un dialogue fécond avec les praticiens de l'urbanisme?

Dans le contexte actuel de mondialisation des recherches, la description micro locale urbaine constitue un défi de taille. Cependant, les notions de quartier, de rue voire d'espaces publics ne sont pas toujours comparables d'un pays à l'autre. Mais le sont-elles aussi dans la même langue? Les terrains de la recherche urbaine sont fortement structurés par l'usage que font de la langue les différents locuteurs qu'ils soient professionnels, experts ou simples habitants. Le terme de Quartier, nous rappelle l'Aventure des mots de la ville (Coudroy de Lille et al., 2010), a exprimé d'abord une réalité défensive et administrative avant d'être mobilisé par les urbanistes et les habitants. S'il correspond traditionnellement au pouvoir de police et à des professions particulières (commerces, marchés et médecins de quartier), son renouveau est issu tant de la recomposition sociale et urbaine des quartiers centraux des villes européennes que de la politique de la ville et des quartiers sensibles mis en œuvre dès le début des années $1980^{2}$. Cependant ces débats n'ont-ils pas une dimension particulière dans l'espace francophone? Si parler, penser (ou écrire) dans une langue n'a guère toujours de vrai équivalent dans une autre (Hagège, 1996), le terme de quartier qui s'associe fortement aux histoires de ville et aux processus de peuplement, à l'héritage de la mémoire collective et à l'identité territoriale des groupes sociaux peut être déplacé en Amérique du Nord, en Afrique du Nord et dans une partie de l'Asie francophone. Nous pouvons en dire de même des termes de rues, places et espaces publics.

Dans cet article nous faisons l'hypothèse que ce lexique, loin d'être isolé de toutes autres influences linguistiques extérieures, incite les études urbaines à des types de récits et des arguments relativement homogènes que l'on peut corréler à l'évolution des représentations des quartiers populaires (I) et aux manières d'organiser l'espace commun (II), ce qui a des implications sur les projets urbains contemporains (III).

4 Pour ce faire, nous proposons au lecteur une revue raisonnée de la littérature francophone principalement centrée sur la seconde moitié du vingtième siècle mais tâchant de repérer les ramifications, courants de pensée et écrits fondamentaux opérant dans la discipline des bifurcations essentielles. Cette histoire intellectuelle des idées nous conduit à tenter de réaliser à la fois une historiographie de chaque notion (quartier, rue, espace public) et une observation de leurs usages contemporains à l'aune des préoccupations sociétales et urbanistiques du moment.

5 Ce propos nous semble fondamental car il questionne la manière dont la tradition monographique permet aux enseignants-chercheurs en école d'architecture et dans les instituts d'urbanisme d'interroger les nouvelles formes d'intervention spatiale et de développer un dialogue fécond et fructueux avec les chercheurs en études urbaines locuteurs d'autres langues que la leur.

\section{Du quartier communauté au quartier recomposé}

Les études de quartier sont certainement plus anciennes que les études urbaines. A la fin du XIXème siècle, les "sociological survey " proposés par Patrick Geddes (1915), et inspirés des enquêtes hygiénistes de Villermé (1840) et Le Play (1879) ont certainement fondé la méthode. Elles reposent sur des inventaires méthodiques des équipements, industries, commerces et habitations. De même au début du XXème siècle les enquêtes 
des géographes tels Vidal de la Blache et Max Sorre ont permis de spatialiser les données sur l'habitat en mettant en relation les formes d'organisation de l'espace et les ressources du territoire (Fijalkow et Lévy, 2008). Vers 1950, les sociologues d'Économie et Humanisme (Astier et Laé 1991; Valladares 2005), et de l'École d'Uriage (Frey, 2000), qui ont contribué à la prise en compte des modes de vie annoncent les premières enquêtes de sociologie urbaine.

7 Ces recherches ont constitué des éléments précurseurs aux études locales qui, après les études rurales d'Edgar Morin (2013) et d'Henri Mendras (1970) ont permis de comprendre la modernisation de la société française, les effets de la croissance urbaine, la transformation de l'habitat et la recomposition des appartenances. Certaines monographies ont consisté à juxtaposer des études sur le site, sur le bâti, sur les activités, sur les fonctions, sans aborder le lien entre ces éléments et une problématique d'ensemble. D'autres ont étudié plus attentivement la démographie, les modes d'organisation familiale, la transmission des biens. À ce renouveau, la sociologie et la géographie urbaine ne sont pas étrangères. Il suffira d'évoquer ici les recherches de Chombart de Lauwe (1952) sur les quartiers ouvriers de l'agglomération parisienne construits dans l'avant-guerre et sur les nouvelles cités d'habitat social (HLM) des années 1950, ainsi que l'analyse de leur disparition par Henri Coing (1966). Ses recherches sur la dilution du quartier ouvrier et parisien du XIIIème coïncident avec le regard nostalgique de Louis Chevalier (1977) sur les quartiers des classes laborieuses et dangereuses. Mais alors qu'Henri Coing s'interroge sur la moyennisation de la classe ouvrière emportée par la rénovation urbaine de l'habitat, Louis Chevalier envisage surtout la transformation du paysage parisien « traditionnel » et l'avènement d'une métropole d'étrangers.

8 Ainsi le recours à l'étude locale a souvent permis de problématiser des questions plus larges et de "penser par cas» les projets urbains affectant les quartiers et autres morceaux de ville. Les monographies ont aussi structuré l'important programme d'Observation $d u$ changement social ${ }^{3}$ qui a permis d'envisager les problématiques émergentes de la fin des années 1980 sur la recomposition des relations sociales en milieu urbain et la participation des habitants,. Le courant anthropologique, attentif aux modes de faire et de dire au quotidien (de Certeau, 1980; de la Pradelle, 1996) et se confrontant aux règles et aux institutions (Anselme, 2000) s'est beaucoup nourri de ces démarches. Il a permis de relativiser la dimension fonctionnelle du quartier et de souligner sa dimension affective (Noschis (1984), de mettre en exergue la notion géographique de "quartier perçu et vécu » (Metton et Bertrand, 1974) et de rejoindre l'écrivain Georges Perec (1974), qui soulignait déjà l'idéologie du quartier ancien des grandes villes comme Paris supposé attractif, nostalgique et convivial.

9 Ces observations ont beaucoup animé les débats sur le déficit de démocratie urbaine (Ledrut, 1969), la critique de la modernité et le droit à la ville (Lefebvre, 1968). Elles ont conduit, dans les années 1970, à une dénonciation de la connotation idéologique et culturaliste de la notion de quartier (Castells, 1975). Selon cette perspective relativiste et structuraliste la focalisation excessive sur les territoires masquait les déterminants relevant de l'ensemble du mode de production capitaliste de l'espace urbain (Castells, 1975).

10 Entre ces oppositions, la critique méthodologique de Bourdieu $(1980,1983)$ sur les « effets de lieux» a aussi relativisé le poids des facteurs spatiaux en comparaison des processus de différenciation sociale. Néanmoins, si le quartier est le lieu d'expression des inégalités, il révèle les contradictions du capitalisme dont il est une échelle de 
compréhension. Ceci conduit à interroger le quartier comme un problème voire une énigme. Les chercheurs qui s'attachent à en expliquer les formes spatiales visibles (leur formation, leur utilisation par les habitants), s'intéressent aussi bien aux interactions entre les usagers qu'aux décisions politiques concernant leur lieu de vie. Ascher $(1995,1998)$ a d'ailleurs souligné combien les élus locaux sont attachés à la notion de quartier alors qu'elle serait dépassée par le développement des mobilités résidentielles et quotidiennes au sein des métapolis du XXIème siècle.

11 Ainsi, régulièrement, les études urbaines francophones remettent en question la pertinence de la notion de quartier ou s'attachent à réhabiliter cette " échelle d'analyse " (Grafmeyer, 2007). Les bonnes raisons pour s'échapper de ce cadre prégnant sont nombreuses, notamment les mobilités quotidiennes et la multiplication des espaces sociaux. Selon Grafmeyer, les trente dernières années de sociologie urbaine ont considéré le quartier, soit comme " une portion de ville », soit comme " un espace de proximité », soit comme un «milieu de vie ", soit comme un « cadre d'action ». Grafmeyer considère que le quartier est porteur d'effets d'autant plus tangibles que ceux-ci s'appuient sur des représentations sociales associées aux quartiers populaires ou aux " grands ensembles » et autres « quartiers sensibles». Or, comme le note Topalov (2003), c'est bien la notion de quartier populaire qui permet de rendre compte des politiques urbaines de restructuration tant en France, qu'au Royaume-Uni et aux Etats-Unis. Par les représentations qui lui sont associées, le quartier offre un cadre comparatif à l'ambition d'une sociologie des villes, d'Europe et d'Amérique du Nord.

12 Cette réponse fondée sur les effets sociaux des catégorisations spatiales est sans doute caractéristique de la sociologie du début des années 2000. Abordant la ville comme une ressource matérielle et symbolique pour ses habitants, elle s'intéresse aux représentations qui fondent leurs pratiques. Dans un souci de dépassement de la critique structuralo-marxiste de l'Ecole de Chicago, « une idéologie urbaine » selon Castells (1975), et de la redécouverte de ses écrits canoniques - les textes de Simmel sur la figure de l'étranger (1902) et ceux de Louis Wirth (1925), enfin traduits en 1979 et 1980 - la sociologie urbaine persiste dans sa lecture politique des processus urbains. L'étude de la ségrégation et l'observation des mobilisations sociales la conduit à renouveler son approche de la notion de proximité spatiale et sociale (Piolle, 1979).

13 Dans ce cadre, l'approche par les usages des quartiers s'avère heuristique. Authier décrit les pratiques sociales de populations relevant d'itinéraires et de projets résidentiels variés dans les vieux quartiers de Lyon, Montpellier, Montreuil et Paris (Authier, 2008). Il discute la thèse ancienne de Simmel selon laquelle la métropole ne se développe pas sur la base de la proximité. Selon ses différentes enquêtes auprès de panels très diversifiés, «le quartier des citadins revêt des configurations plurielles qui s'inscrivent dans des manières de cohabiter et de vivre en ville socialement et spatialement différenciées " (p 23). Ainsi, des habitants qui ont un usage « traditionnel » du quartier comme espace de proximité en côtoient d'autres qui « ont une vie de quartier détachée des pesanteurs du voisinage, et pour qui le quartier fonctionne au contraire comme un espace ouvert sur le «cosmopolitisme» de la vie urbaine». Ces usages s'expliquent autant par les caractéristiques sociales des individus que par leurs situations résidentielles et les caractéristiques de leurs quartiers, c'est-à-dire in fine selon leurs représentations de leur quartier. Ces enquêtes soulignent l'importance du quartier, en tant que cadre de référence, lieu d'attachement, espace de pratiques et de relations, éventuellement compatibles avec une forte mobilité urbaine. Les habitants les plus enclins à entretenir 
des relations à proximité de leur domicile sont ceux qui ont aussi des pratiques et des relations à l'extérieur du quartier et en sont moins dépendants. Dans ce cadre, les usages $\mathrm{du}$ quartier sont fortement polarisés entre ceux qui se réfèrent au logement et au voisinage selon le cadre des sociologues de l'École de Chicago, et ceux qui sont polarisés par les «sociabilités externes" de la ville. Dès lors, si le "quartier-communauté " d'autrefois n'est plus, c'est du côté du sens que les individus lui donnent qu'il faut rechercher sa réalité. Ceci corrobore les efforts des historiens qui relativisent la notion de quartier populaire (Faure, 1993), mais soulignent l'existence de réseaux sociaux structurant des bassins de vie relationnels plus larges que le quartier vécu autour de la famille, du travail et des déplacements (Prost, 1998).

14 Cependant, Michel Agier (1999) souligne l'impact du regard des scientifiques sur le quartier. Il met volontiers à distance les théories urbaines de l'écologie de Chicago en insistant sur leurs dimensions et implications politiques ainsi que sur leur vision de la ville. Comme anthropologue urbain, il se penche sur les « cadres sociaux et territoriaux imposés par le développement fragmentaire de la non-ville» et entreprend une déconstruction de sa propre discipline. Pour Agier (1999), « la conception de la ville en termes de mosaïques de quartiers, de communautés et de ghettos, sans véritable communication ni mélange, sans possibilité d'espaces communs, rendant la synthèse urbaine plus difficile » (p. 58) n'est qu'un postulat. Celui-ci reposerait sur « la croyance en l'existence d'une identité culturelle des pauvres » qui naturaliserait les identités. Agier s'oppose à la notion de «région morale » développée par l'Ecole de Chicago car elle lui semble légitimer une ségrégation supposée spontanée. Il propose plutôt de s'attacher aux processus historiques et imaginaires, comme il le montre par l'usage des termes relatifs à l'habitat au Togo et par ricochet dans les banlieues populaires françaises. Considérant que la ville participe du processus d'ethnicisation, associant communauté, identité et territoire, son programme d'anthropologue consiste à systématiser «l'observation des idéologies de la fragmentation » comme sources de « la non-ville».

15 A cet égard, l'étude du "Ghetto » contemporain par Didier Lapeyronnie (2008) est riche d'enseignements. Comme sociologue mobilisant la notion d'aire morale développée par Wirth (1925), Lapeyronnie décrit une enclave d'habitat social dans une ville moyenne française frappée par la crise économique, avec ses propres règles de fonctionnement, de rapports entre les populations plus ou moins exclues du monde social, de relations genrées, de rupture avec les équipements de l'Etat. Or, bien que le quartier décrit par Lapeyronnie soit homogène socialement et spatialement, la communauté qu'il décrit n'est qu'une communauté éclatée, difficilement mobilisable car minée par la pauvreté.

Ces différentes approches du quartier montrent combien les études urbaines se sont fortement distanciées dans les années 2000 de la notion de communauté populaire. Les débats traditionnels opposant depuis le début du siècle ruralité et urbanité, quartier et agglomération, communauté et société (Tönnies, 1922; Weber, 1921) ne permettent plus de rendre compte du sens donné aux lieux. Certes, la théorie de l'étranger dans la grande ville fondée par Simmel (1902) souligne l'intérêt d'une représentation intégrative du quartier et sa palette de socialités et de spatialités (Bourdin, 1984). Mais d'autres récits sur les quartiers urbains apparaissent dans les études urbaines francophones : aux vieux quartiers populaires d'avant-guerre, succèdent des quartiers d'habitat social fortement ethnicisés et des quartiers gentrifiés de centre-ville qui prennent la place des premiers. Le sens du quartier se fonde désormais sur une transformation de la notion de proximité et d'un nouveau rapport au local, autorisant toutes sortes de communautés sociales, 
ethniques, religieuses et même virtuelles, se dotant éventuellement de compétences politiques. Pour autant, comme le montre Roncayolo (2002), ces différents sens ne se recouvrent pas au cours du temps, mais se juxtaposent dans les usages du quartier. Il est donc temps de rechercher ce qui, dans ces pratiques, fonde le collectif.

\section{L'espace commun : une nouvelle écologie}

17 L'intérêt des chercheurs pour l'espace du quotidien dans son ensemble, notamment les rues et espaces publics s'explique par l'accessibilité de ces espaces à l'observation, permettant de montrer ce qui, concrètement, fait lien entre les acteurs du territoire. L'attention des chercheurs urbains à la notion de rue est récurrente dans le champ. Non seulement elle emprunte à ce que Choay (1979) qualifie de courant culturaliste, mais elle est aussi alimentée par les historiens poursuivant une lecture de la qualité sensible de la rue notamment sonore (Farge, 1979). En analysant le retour à la rue, Éric Charmes (2005), décrit l'histoire doctrinale de l'anti fonctionnalisme et de l'anti modernisme, comme un projet social et spatial. A la suite de Jane Jacobs et The Death and Life of Great American Cities (1961), on assiste à une reconnaissance dans les milieux professionnels comme dans l'imaginaire citadin des valeurs de la rue, valeur paysagère, valeur culturelle et sociale. La rue comme lieu de vie a valeur de "convivialité ». Selon Charmes (2005), ce retour à la rue a constitué un système de sens et de valeurs favorable à la transformation des quartiers populaires et notamment des anciens faubourgs.

Ossatures des villes, lieux des premiers métiers urbains et terrains pionniers de l'exercice de la planification, les rues, boulevards et autres avenues sont alors identifiés comme des terrains privilégiés d'enquête. Les sociologues qui s'intéressent à la poétique de la ville y sont aussi naturellement sensibles (Sansot, 1973) comme les théoriciens de l'ambiance (Augoyard, 2011). Dans les années 1980, les notions de rues et de quartier se soumettent ainsi à celle d'urbanité (Noschis, 1984) qui désigne la qualité des lieux où les relations sociales sont plus souvent acquises voire choisies qu'imposées (Hannerz, 1983; Lévy, 1999).

La scène des quartiers s'attache d'abord à une microsociologie du quotidien (De Certeau, 1980), attentive aux gestes, aux manières et aux expressions qui expriment une identité locale. Ceci renforce la dimension anthropologique et les démarches de type ethnographiques. Dans les années soixante, la redécouverte de la seconde Ecole de Chicago représentée par Goffman (1973), Becker (1963), Strauss (1990) s'inscrit dans ce processus (Grafmeyer, Joseph, 1979). Selon Isaac Joseph, auteur du Passant Considérable et traducteur introducteur (avec Yves Grafmeyer) de l'Ecole de Chicago en France, l'œuvre d'Erving Goffman s'inscrit pleinement dans l'écologie urbaine développant une conception de l'espace public contemporain. « L'espace n'est ni une enveloppe, ni un vide dans lequel prend place un drame ou une intrigue. C'est un milieu plein dans lequel l'activité d'adaptation et de coopération des individus et des collectifs y trouve ses ressources" (Joseph, 2004, p. 130). Ainsi, "La sociologie de Goffman, nous aide à comprendre ce qui nous arrive (...) : confrontées à la pluralité des mondes sociaux nos compétences sociales se mesurent à la capacité que nous avons de parvenir à un accord. C'est ce domaine de l'organisation sociale des rencontres que la microsociologie construit comme un domaine de plein droit » (Joseph, 2004, p.132). Il propose donc une sociologie des civilités et de la société de services, et une approche dramaturgique des faits sociaux, qui permet de comprendre «à quelles conditions spatiales, organisationnelles, 
communicationnelles, la question du civisme ordinaire pouvait être réactivée » (Joseph, 2004, p.132). Il introduit à une sociologie des opportunités spatiales (« des prises») que le public peut trouver dans l'espace et le milieu urbain pour développer son action et se protéger de celles des autres (Joseph, 1997). Le quartier, la rue et l'espace public sont les terrains de ces scènes observées par le chercheur : files d'attente, campements de sans domicile, transports publics, guichets.

Les propositions théoriques de ce courant se traduisent par des orientations méthodologiques moins attentives aux discours des acteurs qu'à leurs pratiques concrètes dans les espaces publics de transport et de mobilité (Joseph, 1999), de soin et de commerce (Péretz, 1992). Le recours et l'attrait de l'ethnométhodologie (Garfinkel, 1986) conduit à un renouvellement des méthodes pour mieux enregistrer l'action et développer des observations situées comme les promenades commentées (Grosjean et Thibaud, 2001). Cette attention à l'intersubjectivité située dans l'espace conduit d'une part à une approche phénoménologique et auto réflexive et d'autre part au courant pragmatiste américain qu'Isaac Joseph (2003) et Hans Joas (2002) associent à la tradition de l'école de Chicago. Elle construit les notions d'espace public et de public autour de l'interprétation, de l'interpellation et les multiples formes de négociation (Joseph, 2003). Prenant pour point de départ les processus de l'action collective, le pragmatisme s'intéresse à la manière dont «l'action collective se heurte à des problèmes et conduit à des conséquences inattendues ou involontaires, qui doivent être traitées de manière réflexive par la collectivité agissante." (Joas, cité par Cefaï et Joseph, 2002, p. 19). Cette proposition s'inscrit dans la philosophie politique de John Dewey, attentive à la manière dont un " public » construit sa propre action collective, s'établit comme une communauté afin de communiquer avec les acteurs affectés et concernés par les conséquences de l'action.

21 Ainsi, l'ouvrage sur les conflits d'urbanité, dirigé par Cefaï et Joseph (2002), s'intéresse au public comme «champ normatif qui agence des dispositions et des disponibilités, des compétences et des formes de confiance constitutives d'une intelligence contextuelle ou mondaine". Le livre, qui parcourt les conflits urbains de Buenos Aires, de Saint-Etienne, de Caracas et de Belleville (entre autres), étudie les formes d'argumentation et d'action sur les conflits d'urbanité comme formes de la vie démocratique.

Parmi de nombreuses applications de ces principes théoriques et méthodologiques, on relèvera aussi deux études de terrains significatives de ce courant de pensée. Dans le quartier parisien de la Goutte d'or, la description des intersubjectivités et des échanges dans l'espace public entre habitants, commerçants, passants et forces de l'ordre conduit à définir des formes de régulation de l'espace public, des agencements réciproques et une ambiance de quartier (Milliot, 2015). A la Nouvelle Orléans, face à la catastrophe naturelle, Stéphane Tonnelat (2012) relève les interactions et les positionnements face au risque qui apparaît alors comme un "problème public ». Cette problématique introduit une réflexion sur l'environnement personnel et les aménités spatiales conduisant à une exploration de la dimension sensible de l'espace urbain et aux rythmes urbains diurne et nocturne (Gwiazdzinski, 2012).

En définitive, pour ce courant de l'écologie urbaine, le quartier et la rue sont moins des espaces géographiques que des lieux communs positionnant les collectifs et les individus. Par exemple, l'espace de la rue peut être un cadre facilitant les interactions multi ethniques et les recompositions sociales liées à la gentrification des quartiers populaires (Simon, 1997). De même, l'étude des cohabitations dans l'espace public et les usages de l'espace se nourrissent d'une ethnographie des réseaux élargissant le prisme de 
l'observation. Ainsi l'étude des parcours migratoires qui réinscrit l'analyse des interactions dans les quartiers, comme à Belzunce par Alain Tarrius (2002), permet de réinterroger deux axes de l'Ecole de Chicago: la notion d'urbanité et les facteurs de coalition des populations dans les territoires. En portant l'attention de l'individu à ses trajectoires, le quartier apparaît comme le reflet d'un monde de réseaux transnationaux (Tarrius, 2008). Le chercheur développe une observation située dans la rue, notamment auprès d'entrepreneurs ethniques. Le quartier apparaît comme une niche économique (Tarrius, 2008).

24 À cet égard, les sciences sociales sont armées pour dialoguer avec les architectes et planificateurs. C'est ce que montre Laurent Fourchard sur les rues de Lagos à propos du film de Rem Koolhaas sur le même terrain, auquel il reproche l'absence d'une vision conflictuelle et politisée de l'espace (2006, p.70) :

« La rue est tout à la fois partagée et disputée. Elle est un espace négocié : les emplacements limitrophes des habitations et boutiques sont loués, la colonisation des espaces vacants ou classés dangereux (voies ferrées, échangeurs, abords d'autoroutes...) est objet de discussions permanentes et d'accords informels entre une multitude d'acteurs privés (syndicats, associations, industriels) et publics (fonctionnaires du gouvernement local et de l'État, des chemins de fer, de la police (Fourchard, 2006, p.65)

Cependant, la catégorisation sociale des espaces publics s'avère particulièrement délicate quand elle interroge les formes du vivre ensemble. L'article de Jacques Donzelot sur «la ville à trois vitesses» (2004), se présente comme une contribution majeure et largement discutée d'un débat sur la multiplication des formes « d'entre soi » contribuant à la reconfiguration de la vie collective. Trois processus définissent trois «entre-soi » dans lesquels la qualité du quartier se trouve prise : quartier de la relégation, quartier de la périurbanisation, quartier gentrifié. La conclusion semble s'imposer que «La ville ne fait plus société ». Mais quelle « ville»? Quels espaces publics?

En fait, la critique des quartiers péri-urbains anomiques (et de leurs habitants) tient autant à l'examen des processus politiques locaux comme la «clubbisation» de la vie urbaine décrits par Éric Charmes (2011) qu'à l'idéologie de la rue qui est le pendant de la figure idéalisée du village (Charmes, 2005). C'est ce que montre le travail d'Eleonora Elguezabal (2015) sur cinq "gated communities» de Buenos Aires ${ }^{4}$. Celle-ci rend compte des différentes frontières sociales à l'intérieur même de ces quartiers, moins homogènes socialement qu'il y paraît de prime abord. Ces observations fines rejoignent celles de l'équipe de la France des Petits moyens (Cartier et al., 2008) selon lesquelles les petites mobilités sociales s'intègrent aux mobilités résidentielles dans un tissu urbain associant pavillonnaire et grands ensembles occupés par des couches moyennes en ascension sociale. Les espaces du quotidien, notamment la rue et les jardins en façade des maisons individuelles, sont fortement investis de significations sociales.

Ces deux exemples illustrent l'importance des dispositifs spatiaux comme la rue et les espaces publics ainsi que les interprétations réciproques entre les acteurs dans la production des lieux. L'espace public est identifié à un problème collectif et l'environnement à un «bien commun » que des groupes d'individus peuvent améliorer, entretenir ou dégrader selon leurs relations sociales internes (Sampson, 2012). Une certaine sociologie de l'espace qui considère les formes spatiales comme parties prenantes des structures sociales (Giddens, 1973; Löw, 2015) trouve ici droit de cité. Et comme les formes architecturales et urbaines sont envisagées comme des acteurs 
(ou du moins des objets actants), les études urbaines ne sont que plus efficientes pour développer leurs observations critiques des projets dans la perspective d'un « tournant spatial».

\section{Le quartier et la rue : des lieux pour l'observation critique des projets urbains}

Si au cours des cinquante dernières années la sociologie urbaine a dépassé la problématique du quartier pour mettre à jour les différentes échelles de proximité entre les groupes sociaux ainsi que les équilibres micro locaux, elle n'a pas perdu de vue que l'espace est aussi bien un « commun » que le lieu d'expression des pouvoirs. À cet égard, la nouvelle production des espaces urbains dans la ville contemporaine peut être un lieu adéquat d'observation.

Ainsi l'analyse socio-économique des projets a mis en évidence les réseaux d'acteurs. Dans les années 2000, l'objectif est de dépasser la critique urbaine marxiste du processus de production de la ville, souvent posée "hors sol» (Castells et al., 1974), malgré une approche empirique de la fabrique urbaine. Cette posture peut suivre la veine des recherches sur la gouvernance des villes européennes (Le Galès, 1995) et sur la construction du pouvoir local par le projet (Pinson, 2009). Mais elle peut aussi s'inscrire dans une analyse située et pragmatiste d'ethnographie des projets urbains en train de se faire. L'observation des négociations à l'oeuvre dans la fabrique de la ville, la circulation des «bonnes pratiques» (Devisme et al., 2007) permet de mobiliser la théorie de l'acteur-réseau et l'analyse de la controverse. Elle met à jour le rôle des acteurs publics et privés dans la circulation de modèles urbains et la standardisation des formes urbaines produites et de la marchandisation des espaces publics. On peut citer à cet égard, les recherches de Laurent Matthey (2013) sur l'ethnographie d'un projet de paysage, ou celle de Pierre-Arnaud Barthel et d'Eric Verdeil (2008) sur les projets urbains méditerranéens. Cette veine renouvelle l'analyse de la gestion urbaine comme le montre Armelle Choplin (2014) dans son étude sur Nouakchott, où interviennent, comme dans de nombreuses villes du Sud, bailleurs de fonds et ONG. La libéralisation foncière provoque l'apparition de nouvelles formes urbaines et induit une impressionnante spéculation qui permet à la ville d'éradiquer ses bidonvilles et de tenter d'entrer dans la compétition mondiale des villes.

Ces recherches posent la question des modèles qui animent la logique des acteurs : élus, aménageurs, pouvoirs techniques des villes et experts urbains (Carriou et Ratouis, 2014). Elles montrent comment des modèles d'urbanismes tels ceux de la ville durable et des éco quartiers s'établissent à partir de nécessités de débouchés économiques voire de valeurs partagées sur les bonnes pratiques d'habiter (Renauld, 2015).

De plus, une interrogation forte porte sur les références à la notion d'attractivité des villes au sein de la compétition mondiale, supportée depuis les années 1990 par les théories de Florida (2005). On assiste, à l'échelle européenne, à un renforcement du rôle économique des moyennes et grandes villes et des «capitales régionales» qui développent une orientation tertiaire ou de services ainsi que des «externalités positives » (Le Galès, 2003). La métropolisation de ces villes autour d'une économie favorable à la recherche-innovation s'accompagne de l'émergence de villes «flexibles » et " créatives ", capables de développer un environnement architectural et urbain propice à 
une l'implantation résidentielle d'une population de cadres (Veltz, 1996; Davezies, 2012). La construction institutionnelle qui accompagne l'enjeu de ces «avantages métropolitains » (Halbert, 2010) permet à une expertise auprès des collectivités locales de se développer. Cependant elle laisse dans l'ombre les villes moyennes et les centres bourgs (Demazière et al., 2012), et elle reproduit invariablement les mêmes recettes et décors « urbains » fortement inspirés par la notion « d'authenticité » (Zukin, 2010).

La notion d'attractivité qui conduit les projets urbains à développer une forme de reconquête des villes-centres, des faubourgs et des espaces anciennement industriels est aussi un bon exemple. L'espace public créé par l'urbanisme du XXI ${ }^{\mathrm{e}}$ siècle en est l'aboutissement logique. La patrimonialisation touristique, la «muséification», la "piétonnisation", accompagnent la mise en exergue de la ville dite " authentique ». Dans la même veine, la "ville nature" avec ses éco et nouveaux quartiers, acquis à la conscience écologique et aux normes de durabilité, met en scène les mêmes figures d'espaces piétonniers, de places et de trames vertes, de circulations douces, et de bâtiments de verre en apparence hospitaliers (Hajek et al., 2015). Les mêmes recettes d'un urbanisme soucieux de l'image et de l'attractivité sont aussi appliquées aux quartiers de la Politique de la ville.

\section{Conclusions et discussions}

Depuis l'Architecture de la ville d'Aldo Rossi (1966), le quartier, la rue, l'espace public sont des échelles nodales de la ville, au cœur des disputes et controverses doctrinaires. Les études urbaines ont permis de mieux accompagner l'évolution du sens de la localité dans notre modernité en révélant les différentes facettes de la notion de proximité et la manière dont les habitants se dotent de compétences politiques pour gérer le bien commun. De celles-ci ont émergé de nouvelles thématiques notamment autour de l'attractivité du territoire et leurs revalorisations soit patrimoniale, soit écologique.

Cette dynamique s'est produite dans l'espace de la francophonie, grâce à l'usage d'un certain lexique à la fois propice à certains récits et modèles de projets urbains. Les termes de "place », de « rue » et de " quartier » renvoient à des réalités phénoménales qui ont un sens particulier dans les villes françaises dans la mesure où elles invoquent des pratiques et des modes de régulation de l'espace public : place du piéton, de la voiture, du marché de plein vent, des bedeaux et des clochards, etc. Les réflexions des chercheurs ne sont pas étrangères à l'évolution de leur sens au cours des cinquante dernières années : par exemple l'idée que tous participent à la production de l'espace, le promoteur comme l'usager, la volonté de lutter contre l'entre soi, l'ouverture de l'espace public aux usages les plus divers participent de courant d'idées qui se matérialisent dans la ville. Tous ces éléments participent de la diversification des sens de la notion de quartier et de sa charge affective contribuant à donner de l'importance à l'espace public.

Ils démontrent que les discours sur la ville contiennent une part performative conduisant les notions abstraites et les habitudes des citadins à s'inscrire dans le paysage architectural et urbain. En urbanisme, plusieurs chercheurs ont montré le rôle des représentations dans la production de l'espace. Les travaux de Rossi et Vanolo (2011), ont documenté la façon dont « l'image de la ville fait l'objet d'une activité systématique de gouvernement grâce à l'invention du marketing territorial » (Rossi et Vanolo, 2011, p. 30). Comme les processus de branding urbain, les discours des planificateurs peuvent préparer des processus de gentrification (Bacqué et Fijalkow, 2006), des politiques de mixité 
sociale, des projets de démolition et déplacement de populations. Cependant les discours ne sont pas seulement des actes de légitimation liés à des intérêts et développant des argumentaires porteurs de valeurs. Ils sont aussi des récits porteurs de représentations de la réalité, d'un passé et d'un futur possible et envisageable. Comme l'écrit le politologue Roe (1994), on doit être attentif aux catégories de pensée qui définissent les sujets et les objets de l'action. Chaque opération d'urbanisme est une scène racontée dans laquelle interviennent, selon les énonciateurs, des types d'habitants, des types de lieux, des processus.

A cet égard, la notion de performativité, développée par le philosophe Austin dans son ouvrage Comment faire des choses avec des mots (1962), démontre combien la parole permet de faire exister des créations, et de leur donner une véritable existence sociale et spatiale. En urbanisme, il ne suffit pas de montrer que le paysage virtuel d'une smart city, le décor des boutiques d'un quartier, et le paysage pacifié d'un éco quartier illustrent la matérialisation de discours. Il faut expliquer comment les discours, les représentations, les récits fonctionnent comme principes organisateurs de l'espace, à plusieurs échelles. Il s'agit de comprendre comment l'urbanisme en tant que production collective d'acteurs dans lesquels interagissent des chercheurs, des élus, des aménageurs, des habitants mobilise et homogénéise des discours destinés à s'ancrer dans le réel dans une mise en scène (Fijalkow, 2017).

Notre analyse a permis de mettre en exergue l'effet d'agenda mettant à l'ordre du jour des types de sites qui façonnent l'action publique et dirigent le regard des chercheurs. On a pu mettre en parallèle l'actualité des années 1950 avec les quartiers-communautés populaires, celles des années 1960 pour les vieux quartiers anciens de centre-ville, les années 1980 avec les nouveaux quartiers HLM du monde populaire, les années 1990 et leurs quartiers piétonnisés et patrimonialisés, dans les années 2000 des ghettos des banlieues françaises ainsi que les quartiers pavillonnaires périurbains des classes moyennes et populaires.

Chacun de ces sites mis en scène avec le concours des chercheurs et des décideurs relève de types de problèmes publics : l'intégration, le déficit d'image, la ségrégation et la relégation, le manque d'attractivité. Ces récits appellent des réponses à la fois politiques, sociales et urbanistiques. En s'attachant à des micro objets comme la rue, l'espace public et le quartier nous avons voulu montrer comment les réflexions des chercheurs participent de cette production de la ville et partagent avec les praticiens des réflexions convergentes.

\section{BIBLIOGRAPHIE}

AGIER, M. (1999). L'Invention de la ville. Banlieue, township, invasions et favelas, Paris, Éditions des Archives contemporaines, $176 \mathrm{p}$.

ANSELME, M. (2000). Du bruit à la parole. La scène politique des cités, La Tour-d'Aigues, Éditions de l'Aube, $279 \mathrm{p}$. 
ASCHER, F. (1995). Métapolis ou l'avenir des villes, Paris, Odile Jacob, 352 p.

ASCHER, F. (1998). « La fin des quartiers? », dans HAUMONT, N. (dir.), L'urbain dans tous ses états. Faire, vivre, dire la ville, Paris, L'Harmattan, p. 183-201.

ASTIER, I., \& J.-F. LAÉ (1991). « La notion de communauté dans les enquêtes sociales sur l'habitat en France. Le groupe d'Économie et humanisme, 1940-1955 », Genèses, vol. 5, no 1, p. 81-106.

AUGOYARD, J.-F. (dir.) (2011). Faire une ambiance. Actes du colloque international, Grenoble, septembre 2008, Éd. À la croisée, 527 p.

AUSTIN, J (1962) Quand dire, c'est faire, Paris Seuil.

AUTHIER, J.-Y. (2008). « Les citadins et leur quartier. Enquêtes auprès d'habitants de quartiers anciens centraux en France ", L'année sociologique, vol. 58, 2008/1, p. 21-46.

BACQUÉ, M.-H. \& Y. FIJALKOW (2006). « En attendant la gentrification : discours et politiques à la Goutte d'Or (1982-2000) ", Sociétés contemporaines, vol. 3, no 63, p. 63-84.

BARTHEL, P.-A., \& É. VERDEIL (2008). « Experts embarqués dans le tournant financier. Des grands projets urbains au sud de la Méditerranée ", Les Annales de la recherche urbaine, no 104, p. 38-48.

BECKER, H.S. (1963). Outsiders: Studies in the Sociology of Deviance, New York, The Free Press of Glencoe, $179 \mathrm{p}$.

BOURDIEU, P. (1993). « Effets de lieu », dans La Misère du Monde, Éditions de Minuit, p. 159-167.

BOURDIEU, P. (1980). « Eléments pour une réflexion critique sur l'idée de région », Actes de la recherche en sciences sociales, vol. 35, no 1, p. 63-72.

BOURDIN, A. (2003). «Urbanisme et quartier. Ce que nous apprend la rive gauche », Terrain, no 41, septembre 2003, p. 137-148.

BOURDIN A. (1984) Le patrimoine réinventé, Paris, Presses universitaires de France

CARTIER, M., COUTANT, I., MASCLET, O. \& Y. SIBLOT (2008). La France des « petits-moyens ». Enquêtes sur la banlieue pavillonnaire, Paris, La Découverte, 324 p. (Collection « Textes à l'appui »)

CARRIOU, C. \& O. RATOUIS (2014) «Quels modèles pour l'urbanisme durable ? ", Métropolitiques, 25 juin 2014. URL : http://www.metropolitiques.eu/Quels-modeles-pour-l-urbanisme.html

CASTELLS, M. (1975). La question urbaine, Paris, Maspéro, 529 p.

CASTELLS, M., GODARD, F. \& V. BALANOWSKI (1974). Monopolville. Analyse des rapports entre l'entreprise, l'Etat et l'urbain à partir d'une enquête sur la croissance industrielle de Dunkerque, Paris, Mouton, $496 \mathrm{p}$.

CÉFAÏ, D et JOSPEH, I.(Eds.), (2002) L'héritage du pragmatisme. Conflits d'urbanité et épreuves de civisme, La tour d'Aigues, Éditions de l'Aube.

CHARMES, É. (2011). La ville émiettée. Essai sur la clubbisation de la vie urbaine, Paris, PUF, 296 p. (Collection « La ville en débat »)

CHARMES, É. (2005). « Le retour à la rue comme support de gentrification », Espaces et sociétés, 2005/3, no 122, p. 115-135.

CHEVALIER, L. (1977). L'Assassinat de Paris, Paris, Calmann-Lévy, 291 p.

CHOAY, F. (1979). L'urbanisme, utopies et réalités. Une anthologie, Paris, Seuil, 446 p. 
CHOMBART DE LAUWE, P.-H. (1952). Paris et l'agglomération parisienne. Tome $1:$ « L'espace social dans une grande cité », 262 p. Tome $2:$ «Méthodes de recherches pour l'étude d'une grande cité », 109 p. Paris, PUF.

CHOPLIN, A. (2014). « Nouakchott : ériger des tours et éradiquer les bidonvilles, ou comment faire rentrer dans la compétition les périphéries du monde ", dans LE BLANC, A., PIERMAY, J.-L., GERVAIS-LAMBONY, P., GIROUD, M., PIERDET, C. \& S. RUFAT (Ed.), Métropoles en débat : (dé)constructions de la ville compétitive, Paris, Presses Universitaires de Paris Ouest, p. 255-272. COING, H. (1966). Rénovation urbaine et changement social. L'̂̂lot no 4, Paris 13e, Paris, Éditions Vie Ouvrière, 296 p.

COUDROY DE LILLE, C., TOPALOV, C., DEPAULE, J.-C. \& B. MARIN (dir.) (2010). L'Aventure des mots de la ville, à travers le temps, les langues, les sociétés, Paris, Robert Laffont, $1568 \mathrm{p}$.

DAVEZIES, L. (2012). La crise qui vient : la nouvelle fracture territoriale, Paris, Seuil, 128 P.

DE CERTEAU, M. (1990). L'invention du quotidien. Tome 1 : «Arts de faire », 375 p. Tome 2 : « Habiter, cuisiner », 316 p. Éd. établie et présentée par Luce Giard, Paris, Gallimard, (1re éd. 1980).

DE LA PRADELLE, M. (1996). Les vendredis de Carpentras. Faire son marché en Provence ou ailleurs, Paris, Fayard, $374 \mathrm{p}$.

DEMAZIÈRE, C., SERRANO, J. \& D. VYE (2012). « Les villes petites et moyennes et leurs acteurs : regards de chercheurs. » Norois, no 223, p. 7-10.

DEVISME, L., DUMONT, M. \& E. ROY (2007). « Le jeu des « bonnes pratiques » dans les opérations urbaines, entre normes et fabrique locale », Espaces et sociétés, 2007/4, no 131, p. 15-31.

DONZELOT, J. (dir.) (2004). « La ville à trois vitesses. Gentrification, relégation, périurbanisation », Esprit, no 3-4, mars-avril 2004, p. 14-39.

DUHAU, E., \& A. GIGLIA (2012). « De la ville moderne aux micro-ordres de la ville insulaire. Les espaces publics contemporains à Mexico », Espaces et sociétés, 2012/2, no 150, p. 15-30.

ELGUEZABAL, E. (2015). Frontières urbaines. Les mondes sociaux des copropriétés fermées, Rennes, PU Rennes, 248 p. (Collection « Géographie sociale »)

FARGE, A. (1979). Vivre dans la rue à Paris au XVIIIe siècle, Paris, Gallimard, 1992 (1ère éd. 1979), $256 \mathrm{p}$.

FAURE, A. (1993). «Le local : une approche du quartier populaire (Paris 1880-1914) », Mélanges de l'école française de Rome, 105(2), p. 489-502.

FIJALKOW, Y. (dir) (2017). Dire la ville c'est faire la ville, Villeneuve-d'Ascq, Presses Universitaires du Septentrion, $194 \mathrm{p}$.

FIJALKOW, Y. \& J.-P. LÉVY (2008). « Un siècle d'étude sur l'habitat français en géographie urbaine (1900-2000) ", Annales de géographie, 2008/4, no 662, p. 20-41.

FLORIDA, R. (2005). Cities and the Creative Class, London, Routledge, $208 \mathrm{p}$.

FOURCHARD, L. (2006). « Les rues de Lagos : espaces disputés/espaces partagés », Flux, 2006/4, no 66-67, p. 62-72.

FREY, J.-P. (2000). « Paul-Henry Chombart de Lauwe : la sociologie urbaine française entre morphologie et structures », Espaces et sociétés, 2000/3, no 103, p. 27-56.

GARFINKEL, H. (1986). Ethnomethodological Studies of Work, Londres, Routledge \& Kegan Paul, 208 p. 
GEDDES, P. (1915) City in evolution, London, Williams and Norgate Ltd

GIDDENS, A. (1973). The class structure of the advanced societies, London, Hutchinson, 339 p.

GOFFMAN, E. (1983) La Mise en scène de la vie quotidienne. Tome $1:$ « La Présentation de soi », 256 p. Tome $2:$ « Les relations en public », 368 p. Paris, Éditions de Minuit (Collection « Le Sens Commun »)

GRAFMEYER, Y. (2007). « Le quartier des sociologues », dans AUTHIER, J.-Y., BACQUÉ, M.-H. \& F. GUÉRIN-PACE (dir.), Le quartier, Paris, La Découverte, p. 21-31.

GROSJEAN, M. \& J.-P. THIBAUD (2001). L'espace urbain en méthodes, Marseille, Editions Parenthèses, $217 \mathrm{p}$.

GWIAZDZINSKI, L. (2012). « Un possible manifeste. Éloge de l'errance et de la désorientation », dans DÉSANGES, G. \& H. GUENIN (Ed.), Erre. Variations labyrinthiques, Metz, Éditions Le Centre Pompidou-Metz, p. 52-56.

HAGÈGE, C. (1996). L'Homme de paroles : contribution linguistique aux sciences humaines, Paris, Fayard, 324 p.

HAJEK, I., HAMMAN, P. \& J.-P. LÉVY (2015). De la ville durable à la nature en ville, Villeneuve-d'Ascq, Presses universitaires du Septentrion, $318 \mathrm{p}$.

HALBERT, L. (2010). L'avantage métropolitain, Paris, PUF, 152 p. (Collection « La ville en débat »)

HANNERZ, U. (1983). Explorer la ville. Éléments d'anthropologie urbaine, Paris, Les Éditions de Minuit, $432 \mathrm{p}$.

JACOBS, J. (1961). The Death and Life of Great American Cities, New York, Random House, 458 p.

JOAS, H. (2002). « Pragmatisme et sciences sociales. L'héritage de l'école de Chicago », dans CEFAÏ, D. \& I. JOSEPH (Ed.), L'héritage du pragmatisme. Conflits d'urbanité et épreuves de civisme, La Tour d'Aigues, Éditions de l'Aube, p 17-49.

JOSEPH, I. (dir.) (1999). Villes en gare, La Tour d'Aigues, Éditions de l'Aube, 309 p.

JOSEPH, I. (1984). Le passant considérable. Essai sur la dispersion de l'espace public, Paris, Librairie des Méridiens, 146 p. (Collection « Sociologie des formes »)

JOSEPH, I. (1997). « Prises, réserves, épreuves », Communications, no 65, p. 131-142.

JOSEPH, I. (2003). « La notion de public : Simmel, l'écologie urbaine et Goffman », dans CEFAÏ, D \& D. PASQUIER (Ed.), Le sens du public, Paris, PUF, p. 329-346.

JOSEPH, I. (2004). « Goffman et l'écologie urbaine », Les Annales de la recherche urbaine, no 95, p. 130-133.

KAUFMANN, V. (2008). Les paradoxes de la mobilité, bouger, s'enraciner, Lausanne, Presses polytechniques et universitaires romandes, $115 \mathrm{p}$.

KOOLHAAS, R. (2011). « Bigness ou le problème de la grande dimension », dans Junkspace.

Repenser radicalement l'espace urbain, Paris, Payot, p. 29-42.

LACAZE, J.-P. (2012). Les méthodes de l'urbanisme, Paris, PUF, 2012 (6e éd.), 127 p. (Collection « Que sais-je?»)

LAPEYRONNIE, D. (2008). Ghetto urbain. Ségrégation, violence, pauvreté en France aujourd'hui, Paris, Robert Laffont, 630 p. (Collection « Le monde comme il va »)

LE GALÈS, P. (2003). Le retour des villes européennes. Sociétés urbaines, mondialisation gouvernement et gouvernance, Paris, Presses de Sciences Po, $454 \mathrm{p}$. 
LE GALÈS, P. (1995). « Du gouvernement des villes à la gouvernance urbaine. » Revue française de science politique, 1995/1, vol. 45, p. 57-95.

LE PLAY, F. (1879) Les ouvriers européens : étude sur les travaux, la vie domestique et la condition morale des populations ouvrières de l'Europe, Tours, Mame et fils, 6 vol.

LEDRUT, R. (1968). Sociologie urbaine, Paris Presses Universitaires de France, 224 p.

LEFEBVRE, H. (1968). Le droit à la ville, Paris, Anthropos, 164 p.

LÉVY, J. (1999). « Penser la ville », dans LÉVY, J. (Ed.), Le tournant géographique. Penser l'espace pour lire le monde, Paris, Belin, $400 \mathrm{p}$.

LÉVY, J. (2009). « Mondialisation des villes », dans STÉBÉ, J.-M. \& H. MARCHAL (Ed.), Traité sur la ville, Paris, PUF, p. 667-721.

LEWIS, O. (1969). La Vida : une famille porto-ricaine dans une culture de pauvreté: San Juan et New York, Paris, Gallimard, 820 p.

LÖW, M. (2015). Sociologie de l'espace, Paris, Maison des Sciences de l'Homme, 302 p.

MATTHEY, L. (2013). « Les faiseurs de paysage. Ethnographie d'un projet urbain », L'Information géographique, 2013/1, vol. 77, p. 6-24.

MENDRAS, H. (1970). La fin des paysans : changement et innovations dans les sociétés rurales françaises, Paris, Armand Colin, 306 p.

METTON, A., \& M.-J. BERTRAND (1974). « Les espaces vécus dans une grande agglomération. » L'espace géographique, vol. 3, no 2, p. 137-146.

MILLIOT, V. (2015). « Remettre de l'ordre dans la rue. Politiques de l'espace public à la Goutte-d'Or (Paris) », Ethnologie française, 2015/3, no 153, p. 431-443.

MISSAOUI, L. \& A. TARRIUS (2006). « Villes et migrants, du lieu-monde au lieu-passage », Revue européenne des migrations internationales, vol. 22, no 2, p. 43-65.

MORIN, E. (2013). La métamorphose de Plozévet : Commune en France, Paris, Fayard / Pluriel, 512 p.

MUMFORD, L. (1961). La cité à travers l'histoire, Marseille, Agone, 2011 (1ère éd. 1961), 922 p.

NOSCHIS, K. (1984). Signification affective du quartier, Paris, Librairie des Méridiens, 170 p.

PAQUOT, T. (2009). L'espace public, Paris, La Découverte, 128 p. (Collection « Repères »)

PEREC, G. (1974). Espèces d'espaces, Paris, Galilée, 124 p.

PÉRETZ, H. (1992). « Le vendeur, la vendeuse et leur cliente. Ethnographie du prêt-à-porter de luxe », Revue française de sociologie, vol. 33, no 1, p. 49-72.

PINSON, G. (2009). Gouverner la ville par projet : urbanisme et gouvernance des villes européennes, Paris, Presses de Sciences Po, 420 p.

PIOLLE, X. (1979). Les Citadins et leur ville. Approche de phénomènes urbains et recherches méthodologiques, Toulouse, Privat, $432 \mathrm{p}$.

PROST, A. (1998) « La rue de la Goutte-d'Or et la rue Polonceau entre les deux guerres ». Le mouvement social, p. 9-27.

RAMADIER, T. (2002). « Rapport au quartier, représentation de l'espace et mobilité quotidienne », Espaces et sociétés, 2002/1, no 108, p. 111-132.

RENAULD, Vincent (2014) Fabrication et usage des écoquartiers. Essai critique sur la généralisation de l'aménagement durable en France. Lausanne, Presses polytechniques et universitaires romandes. 
ROE, E. (1994). Narrative policy Analysis: Theory and Practice, Durham, Duke University Press, 240 p. RONCAYOLO, M. (2002). Lectures de villes. Formes et temps, Marseille, Editions Parenthèses, 386 p. ROSSI, A. (1966). L'Architecture de la ville, Paris, L'Équerre, 1981 (1ère éd. 1966), 295 p. ROSSI, U. \& A. VANOLO (2011). Urban political geographies: A global perspective, London, Sage, 232 p. SAMPSON, R.-J. (2012). Great American city: Chicago and the enduring neighborhood effect, Chicago, University of Chicago Press, $552 \mathrm{p}$.

SANSOT, P. (1973). Poétique de la ville, Paris, Klincksieck, 422 p.

SIMMEL, G. (1902). « La figure de l'étranger », dans GRAFMEYER, Y. \& I. JOSEPH (1979). L'École de Chicago - Naissance de l'écologie urbaine, Paris, Aubier, 335 p.

SIMON, P. (1997). « Les usages sociaux de la rue dans un quartier cosmopolite », Espaces et sociétés, 1997/2, no 90, p. 43-68.

STRAUSS, A. \& J. CORBIN (1990). Basics of Qualitative Research: Grounded Theory Procedures and Techniques. Trad. fr. (2004) : Les fondements de la recherche qualitative. Techniques et procédures de développement de la théorie enracinée, Fribourg, Academic Press Fribourg, 342 p.

TARRIUS, A. (2002). La Mondialisation par le bas. Les nouveaux nomades de l'économie souterraine, Paris, Balland, $169 \mathrm{p}$.

TARRIUS, A. (2007). « Nouvelles formes migratoires, nouveaux cosmopolitismes », dans BASSAND, M., KAUFMANN, V. \& JOYE D. (Ed.), Enjeux de la sociologie urbaine, Lausanne, Presses polytechniques et universitaires romandes, p. 135-160.

TARRIUS, A. (2008). « Migrations en réseaux et cohabitations urbaines aux bordures de l'Europe ", L’Année sociologique, 2008/1, vol. 58, p. 71-93.

THIBAUD, J.-P. (2010). « La ville à l'épreuve des sens », dans COUTARD, O. \& J.-P. LÉVY (Ed.), Ecologies Urbaines, Paris, Économica-Anthropos, p. 198-213.

TISSOT, S. (dir.) (2014). « Les espaces de l'entre-soi », Actes de la recherche en sciences sociales, no 204, Paris, Seuil, 140 p.

TONNELAT, S. (2012). « La dimension sensible des problèmes publics. La plateforme d'observation du bayou et la viabilité d'un quartier de la Nouvelle Orléans », dans CEFAÏ, D. \& C. TERZI (Ed.), L'expérience des problèmes publics, Paris, Éditions EHESS, p. 163-190

TÖNNIES, F. (1977). Communauté et société. Catégories fondamentales de la sociologie pure, Paris, RetzC.E.P.L., 1ère éd. 1922, 285 p.

TOPALOV, C., (2003). Les constructions savantes du quartier (France, Grande-Bretagne, États-Unis), Paris, CSU, $199 \mathrm{p}$.

VALLADARES, L. (2005). « Louis-Joseph Lebret et les favelas de Rio de Janeiro (1957-1959) : enquêter pour l'action ", Genèses, 2005/3, no 60, p. 31-56.

VELTZ, P. (1996). Mondialisation, villes et territoires : Une économie d'archipel, Paris, PUF, 262 p. (Collection "Quadrige »)

VILLERMÉ, L-R. (1971). Tableau de l'état physique et moral des ouvriers employés dans les manufactures de coton, de laine et de soie, Paris, Union générale d'Éditions, (1ère éd. 1840, J. Renouard), 316 p.

WEBER, M. (1921). Économie et Société. Les Catégories de la sociologie. Tome premier, Paris, Plon, 650 p. (Traduction française : 1971, 1995, 2003) 
WIRTH, L. (1925). Le Ghetto, Traduction de P.-J. ROTMAN (1980), Paris, Éditions du Champ urbain, PUG, $308 \mathrm{p}$.

ZUKIN, S. (2010). Naked city: The death and life of authentic urban places, New York, Oxford University Press, $312 \mathrm{p}$.

\section{AUTRES REFERENCES PERTINENTES NON CITÉES DANS LE TEXTE}

BOURDIN, A. (2003). « Urbanisme et quartier. Ce que nous apprend la rive gauche », Terrain, no 41, septembre 2003, p. 137-148.

DUHAU, E., \& A. GIGLIA (2012). « De la ville moderne aux micro-ordres de la ville insulaire. Les espaces publics contemporains à Mexico ", Espaces et sociétés, 2012/2, no 150, p. 15-30.

HALBWACHS, M. (1938). Morphologie sociale, Paris, Armand Colin, 1970 (1 ère éd. 1938), 190 p.

JOSEPH, I. (1984). Le passant considérable. Essai sur la dispersion de l'espace public, Paris, Librairie des Méridiens, 146 p. (Collection « Sociologie des formes »)

KAUFMANN, V. (2008). Les paradoxes de la mobilité, bouger, s'enraciner, Lausanne, Presses polytechniques et universitaires romandes, $115 \mathrm{p}$.

LACAZE, J.-P. (2012). Les méthodes de l'urbanisme, Paris, PUF, 2012 (6éd.), 127 p. (Collection « Que sais-je?»)

LÉVY, J. (2009). « Mondialisation des villes », dans STÉBÉ, J.-M. \& H. MARCHAL (Ed.), Traité sur la ville, Paris, PUF, p. 667-721.

LEWIS, O. (1969). La Vida : une famille porto-ricaine dans une culture de pauvreté: San Juan et New York, Paris, Gallimard, $820 \mathrm{p}$.

MILLIOT, V. (2015). « Remettre de l'ordre dans la rue. Politiques de l'espace public à la Goutte-d'Or (Paris) », Ethnologie française, 2015/3, no 153, p. 431-443.

MISSAOUI, L. \& A. TARRIUS (2006). « Villes et migrants, du lieu-monde au lieu-passage », Revue européenne des migrations internationales, vol. 22, no 2, p. 43-65.

MUMFORD, L. (1961). La cité à travers l'histoire, Marseille, Agone, 2011 (1 ère éd. 1961), 922 p.

PAQUOT, T. (2009). L'espace public, Paris, La Découverte, 128 p. (Collection « Repères »)

PIOLLE, X. (1979). Les Citadins et leur ville. Approche de phénomènes urbains et recherches méthodologiques, Toulouse, Privat, $432 \mathrm{p}$.

RAMADIER, T. (2002). « Rapport au quartier, représentation de l'espace et mobilité quotidienne », Espaces et sociétés, 2002/1, no 108, p. 111-132.

TARRIUS, A. (2007). « Nouvelles formes migratoires, nouveaux cosmopolitismes », dans BASSAND, M., KAUFMANN, V. \& JOYE D. (Ed.), Enjeux de la sociologie urbaine, Lausanne, Presses polytechniques et universitaires romandes, p. 135-160.

THIBAUD, J.-P. (2010). « La ville à l'épreuve des sens », dans COUTARD, O. \& J.-P. LÉVY (Ed.), Ecologies Urbaines, Paris, Économica-Anthropos, p. 198-213.

\section{NOTES}

2. Voir Espaces et Sociétés, no 132-133, 2008.

3. . Entre 1977 et 1981, le CNRS soutient le programme OCS, "Observation continue du changement social et culturel" proposé par Henri Mendras, Jacques Lautman et Odile 
Benoit-Guilbot. Ce programme associe près de 200 chercheurs de plusieurs disciplines autour d'une problématique et d'un objet central : le changement social et le local. Le terrain d'étude est constitué de 60 localités françaises, Les travaux, foisonnants, seront publiés en 2002-2003 dans une collection en 18 volumes, les Cahiers de l'Observation du changement social.

4. Voir le numéro des Actes de la recherche en sciences sociales sous la direction de Sylvie Tissot sur les « espaces de l'entre-soi » (Tissot, 2014).

\section{RÉSUMÉS}

Considérant les études urbaines comme un champ de recherche composite issu de savoirs académiques et de savoirs d'action, cet article propose de retracer la contribution des monographies de villes et de quartiers, mobilisées dès l'origine des études urbaines, aux questions des professionnels de l'urbain. Il convoque l'histoire des notions, décrivant les micro-échelles comme le quartier et la rue, ainsi que celle d'espace public et l'examen des outils théoriques décrivant les processus socio-économiques de production de l'urbain. Dans ce cadre, il s'interroge sur le rôle de la langue française. Il fait l'hypothèse que ce lexique, loin d'être isolé de toutes autres influences linguistiques extérieures, incite les études urbaines à des types de récits et des arguments relativement homogènes que l'on peut corréler à l'évolution des représentations des quartiers populaires (I) et aux manières d'organiser l'espace commun (II), ce qui a des implications sur les projets urbains contemporains (III). L'article présente une revue raisonnée de la littérature francophone de la seconde moitié du vingtième siècle et repère les ramifications, courants de pensée et écrits fondamentaux opérant dans la discipline des bifurcations essentielles. Cette histoire intellectuelle des idées conduit à tenter de réaliser une historiographie de chaque notion (quartier, rue, espace public) et une observation de leurs usages contemporains à l'aune des préoccupations sociétales et urbanistiques du moment.

This paper considers urban studies as a field of composite research based on academic and action knowledge. Il proposes to trace the contribution of monographs of cities and neighborhoods, wrote from the beginning of urban studies, to the questions of professionals. It brings together the history of notions, describing micro-scales such as "neighbourhood" and "street", as well as the notion of "public space". It examines theoretical tools describing the socio-economic processes of urban production. In this context, he questions the role of the French language. It is hypothesised that this lexicon is not isolated from any other outside linguistic influences. But it encourages urban studies to relatively homogeneous types of narratives and arguments that can be correlated with the evolution of representations of working-class neighborhoods(I) and organized common space (II), which has implications for contemporary urban projects (III). This paper presents a reasoned review of the French literature of the second half of the twentieth century and identifies the ramifications, currents of thought and fundamental writings. This intellectual history of ideas leads to an attempt to make a historiography of each notion in the light of the societal and urbanistic concerns of the moment.

\section{INDEX}

Mots-clés : Quartier, rue, espace public, sociologie urbaine, urbanisme 


\section{AUTEUR}

YANKEL FIJALKOW AVEC LA COLLABORATION SCIENTIFIQUE D'AMÉLIE NICOLAS

UMR AAU-CRENAU and École Nationale Supérieure D’Architecture Paris-Malaquais

Centre de Recherche sur l'Habitat

UMR 7218 LAVUE CNRS

Ecole Nationale Supérieure d'Architecture Paris Val de Seine 